(Aus der König1. Frauenklinik in Dresden.)

\title{
Ueber die Zange in der Therapie des engen Beckens zur Rettung des Kindes.
}

Von

\section{Dr. Leisewitz, \\ Volontärassistenten.}

Im Jahre 1896 erschien in diesem Archiv (50. Band, 2. Heft) die Arbeit von Wahl: Ueber die Entbindungen mit der Zange in der kgl. Frauenklinik zu Dresden in den Jahren 1889 bis 1. Januar 1894. Ihr war die Arbeit von Münchmeyer vorausgegangen, die das gleiche Thema für die Jahre 1883-1888 behandelt hatte. An diese beiden Arbeiten soll sich eine abermalige Zusammenstellung der Entbindungen mittels Forceps in hiesiger Klinik anschliessen, die sämmtliche Fälle bis zur Neuzeit, d. h. 1. Januar 1907 berücksichtigt.

In ibr soll die Anlegung der Zange zur Rettung des kindlichen Lebens der leitende Gedanke sein, ohne dass dadurch die anderen Anzeichen, welche bei den Zangenoperationen in Betracht kommen, vernachlässigt werden. Für den Praktiker ist es von grösstem Interesse, hierbei die 5 verschiedenen Beckengruppen (allgemein verengtes, normales, plattes, platt rhachitisches und allgemein verengt plattrbachitisches Becken) erläutert $\mathrm{zu}$ sehen. Sie sollen in ihrer Häufigkeit sowohl im Allgemeinen, als speciell bei hoch- und tiefstehendem Kopfe (Zange im Beckeneingang, in Beckenmitte und im Beckenausgang), ferner in der Anzeigestellung: und in der Prognose für Mutter und Kind gegenüberstellt und kritisch beleuchtet werden.

Bei der grossen Menge pathologischer Geburten, welche wesentlich auf dem hohen Procentsatz an engen Becken beruhen, wird 
diese Gegenüberstellung manche Auseinandersetzung herbeiführen, die zunächst für klinische Verhältnisse, aber nicht für die allgemeine Praxis ihre Gültigkeit besitzt. Sie wird aber den Beweis liefern, dass bei günstigen äusseren Verhältnissen, wie sie die Einrichtungen einer Klinik mit sich bringen, die Indication eine gewisse Spannweite erhalten kann, ohne dass dadurch, einen geübten Operateur vorausgesetzt, Mutter und Kind schwerer geschädigt oder überhaupt geschädigt werden. So kann vor Allem die Indicationsgrenze ziemlich genau gezogen werden, wenn verschiedene Operationsarten bei dem betreffenden Falle nach den jetzt bestehenden geburtshülflichen Lehrsätzen in Betracht kommen; es stellen sich der hohen Zange, die nur noch ganz ausnahmsweise in einzelnen Fällen an hiesiger Klinik gemacht wurde, gegenüber die Wendung und Extraction, die Hebotomie, die Sectio eaesarea conservativa und die Perforation des lebenden Kindes. Die Wendung und Extraction gilt bei Erstgebärenden von Anfang an wegen der grossen Gefahr für das Kind bei dem Durchziehen durch die noch straffen mütterlichen Weichtheile als ausgeschlossen. Bei Mehrgebärenden tritt sie in ganz bestimmten Fällen und bei scharf umgrenzter Beckenenge (siehe Lichtenstein) (bei Nichteintreten des Kopfes nach längerer Geburtsdauer und vollständigem Muttermund) in ihre Rechte und zwar mit oder ohne beckenerweiternde Operation (Hebotomie). Damit wird die hohe Zange ausgeschlossen, die so häufig, aucb selbst in der Hand des geübten Operateurs zu schweren Verletzungen der Mutter und des Kindes führen kann und dadurch nicht den Zweck der Anlegung erfüllt, nämlich die Rettung des kindlichen Lebens. Auch nach einer Hebotomie kommt es, wenn die Wendung und Extraction aus irgend einem Grunde nicht angängig ist, nicht zur hohen Zange, sondern nach Impression des Kopfes zur Zange in Beckenmitte.

Verweilen wir bei diesem Punkte etwas länger ond wägen nach unseren Beobachtungen einen Vergleich mit denen anderer Geburtshelfer $a b$, ob eine Hebotomie oder ein hoher Forceps für die Mutter von grösserem Nachtheil ist (immer eine Beckenverengerung vorausgesetzt, die überhaupt die beiden Operationsarten noch zulässt), so kommt man zu dem Schluss, dass eine Hebotomie mit sich anschliessendem Forceps an dem in Beckenmitte befindlichen Kopf oder mit Wendung und Extraction wegen noch beweglichen Kopfes immer noch die Mutter mehr schützt als die hohe Zange; denn bei den 51 ausgeführten Hebotomien haben wir noch 
keinen Todesfall erlebt, von Seiten des Beckens noch keine nachtheilige Störung bemerkt und haben, die wohl immer noch vorkommenden, manchmal nicht zu vermeidenden Verletzungen der Weichtheile zu guter Ausheilung gelangen sehen (s. Kannegiesser). Die Verletzungen durch hohe Zange aber, die meistens schwere Quetschwunden sind, führen ja, wie in anderen Statistiken zu lesen ist, nicht selten zum Tode. Fast noch wichtiger ist aber die Frage: schädigt die Hebotomie mit nachfolgendem Forceps oder Wendung und Extraction das Kind mehr, bezw. gefährdet sie das Leben desselben mehr als die hohe Zange? Diese Frage wird am besten schon hier mit Zahlen beantwortet auf Grund der Tabelle im Winckel'schen Handbuch, Bd. III, I. Theil, S. 572, welcher die spärliche Zahl unserer hohen Zangen angefügt werden soll:

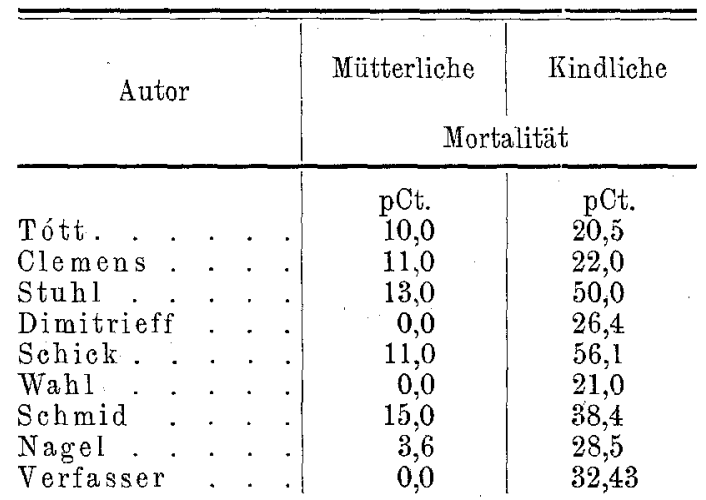

Demnach blieben also bei uns nach hoher Zange nur $67,57 \mathrm{pCt}$. Kinder am Leben, wäbrend nach 51 Hebotomien bei 0 pCt. mütterlicher Mortalität nur 8 pCt. Kinder starben, sonach also 92 pCt. Kinder am Leben blieben. Berücksichtigt man dabei noch, dass die ungünstigen Ausgänge für 4 Kinder nur bei den ersten Hebotomien vorkamen, so kommt man auch hier zu dem Schluss, dass auch für das Kind die Hebotomie von weit grösserem Vortheil ist, als die hohe Zange.

Krönig zieht in seinem Vortrag: "Wie weit soll das Recht des Kindes auf Leben bei der Geburt gewahrt werden" (Vers. d. Naturf. u. Aerzte, Meran) ebenfalls die Folgerung, dass bei engem Becken und aseptischem Geburtskanal eine beckenerweiternde Operation der hohen Zange vorzuziehen sei.

Es mögen nun einige statistische Bemerkungen mit den aus ihnen sich ergebenden Folgerungen im Vergleiche mit andern Kliniken angereiht werden. 
In der Zeit vom 1. Januar 1894 bis zum 1. Januar 1907 wurden in hiesiger Klinik unter 27238 Geburten 697 Zangenoperationen vorgenommen, also $2,55 \mathrm{pCt}$.

In die Statistik, wie sie im Winckel'schen Handbuch vorliegt, würden diese letzten Zahlen als 3. Gruppe an 6. Stelle einzufügen sein.

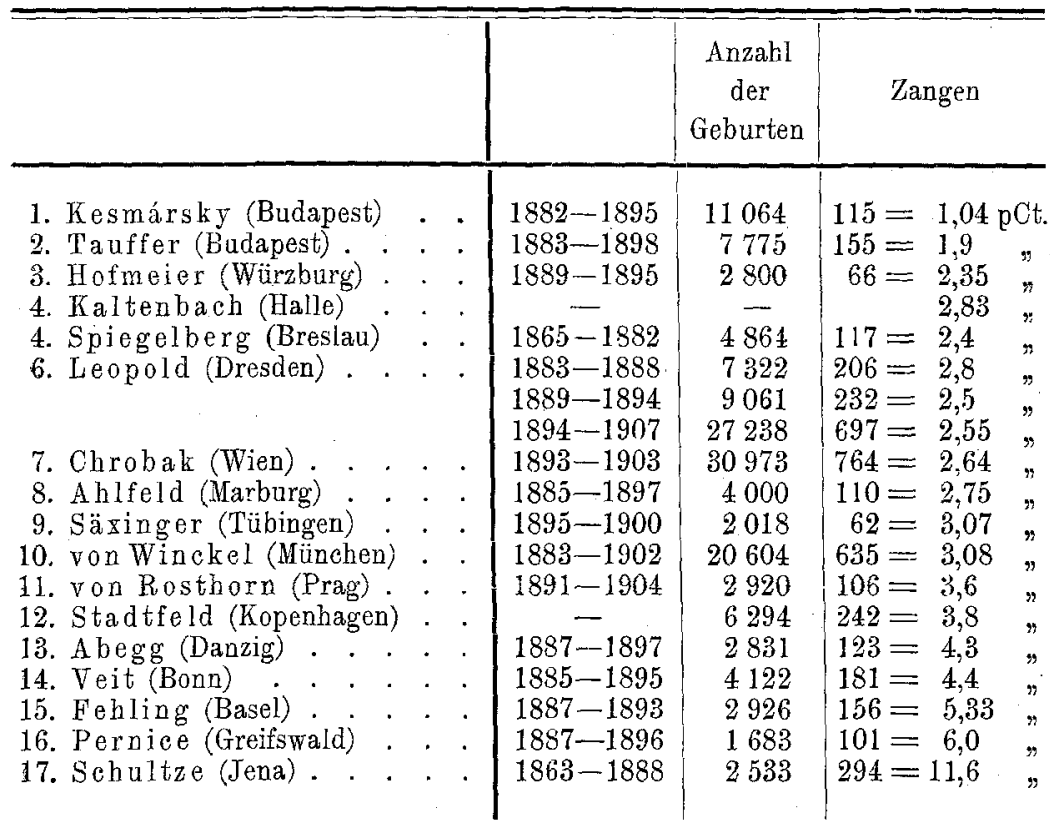

Neu eingeschoben sind hier auch die Resultate der Wiener Klinik von Chrobak; die Zahlen der hiesigen Klinik aus Münohmeyer's und Wahl's Arbeiten sind denen der letzten 13 Jahre gegenübergestellt. Dabei ergiebt sich, dass seit 1894 die Häufigkeit der Zangenanlegung nur um Weniges gestiegen ist, nämlich um 5/100 pCt.

Worauf die grossen Unterschiede in den Zahlen der einzelnen Kliniken liegen, ist hinlänglich bekannt. Sie gründen sich einmal auf das rom engen Becken schwer belastete Material mancher Anstalten. Andererseits wird an Kliniken mit geringem geburtshülflichen Material zur Uebung der Assistenten und Studirenden die Zange mit sehr breiter Anzeigestellung ausgeführt. Und von dieser hängt ja naturgemäss am meisten die Häufigkeit der Zangenentbindungen $a b$.

So gehen vor Allem die Ansichten über die Berechtigung der Zangenanlegung bei der sogenannten Wehenschwäche sehr aus- 
einander. Die Einen, unter ihnen vor Allem von Winckel, greifen in solchen Fällen zur Zange nur dann, wenn gleichzeitig eine ernste Gefahr für Mutter oder Kind besteht, Andere hingegen seben die Nothwendigkeit der Zange schon dann, wenn die Austreibungsperiode zu lange dauert, wenn ein nur langsames Fortschreiten der Geburt oder ein zeitweiser Stillstand derselben festzustellen ist, ohne dass für den Augenblick eine grössere Gefahr für Mutter oder Kind besteht.

In diesem Punkte verhalten wir uns folgendermaassen. Handelt es sich, wie fast immer, um ein enges Becken, namentlich um einen durch vorspringende Spinae ischiadicae, unbewegliches Steissbein und spitzen Schambogen verengten Beckenausgang, wie bei allgemein verengten oder auch allgemein verengten plattrhachitischen Becken, mit Ueberwiegen der allgemeinen Verengerung, so sehen wir in der durch die lange Geburtsdauer in Folge Ueberanstrengung und Uebermüdung entstandenenWehenschwäche eine Gefahr erstens für die Mutter. Denn sie würde keinesfalls, selbst durch eine in Folge von Morphiuminjection hervorgerufene Erbolungspause kaum wieder vollständig zu Kräften kommen, um die grossen Widerstände des Beckenausgangs noch zu überwinden. Zweitens aber vor Allem für das Kind, denn durch die andauernde und lange Zeit währende Compression des kindlichen Schädels zwischen Beckenmitte und Beckenausgang würde, wenn auch nicht schon im Augenblick der Zangenanlegung, so doch sicher nach kurzer Zeit langsam eine Asphyxie eintreten. Sie schädigt den kindlichen Organismus durch zu lange Compression des Kopfes und führt schliesslich zu tödtlicher Hyperämie bezw. Oedem und Blutungen in das Gehirn.

Demnach wägen wir in jedem Falle genau ab, warten aber nur in äusserst seltenen Fällen so lange, bis die Gefahr für Mutter und Kind, oder für eines von beiden ganz auffallend ist, also bis etwa Temperatur- und Pulssteigerung, oder völlige Erschlaffung bei der Mutter eintritt, oder bis bei dem Kinde die Asphyxie durch auffallendes Sinken oder Beschleunigung der Herztöne und rasch wachsende Kopfgeschwolst wahrnehmbar wird. Zeigen sich ja doch schon frühzeitig für den aufmerksamen Beobachter häufig genug ganz feine Zeichen, die die ferne Gefahr ankïnden, z. B. das beginnende Oedem der Muttermundslippen, vor Allem beim Kinde aber die langsame Erholung der Herztöne in der Wehenpause, die beginnende Accentuation des zweiten Tones, der rei- 
bende, manchmal auch dumpf hauchende erste Ton. Bei häufiger Zählung der Herztöne in der Wehenpause wird man dann auch feststellen können, dass Unregelmässigkeiten in der Zahl der Schläge, auch kleine Unterschiede in der Qualität auftreten; so schwer es im Anfang ist, diese feinen Zeichen sofort zu erkennen, so sicher fühlt man sich durch fortgesetzte Uebung im Auscultiren dann in der stricten Indicationsstellung, die eben dann keine allgemeine, sondern eine speziell für jeden Fall gegebene ist.

Diejenigen Kliniken, die vor einer Zangenanlegung bei Wehenschwäche warnen, fürchten die oft schweren atonischen Nachblutungen und sie rathen vollkommen mit Recht dem praktischen Geburtshelfer ab, ohne weitere ernste Indication die Zange anzulegen; denn nicht immer vermag er sofort eine schwere Blutung zu beherrschen, während in der Klinik alle Mittel vorgesehen sind.

Handelt es sich um ein normales Becken und normale Einstellung des Kopfes, und ist nur die Wehenthätigkeit alterirt, so warten allerdings auch wir längere Zeit und lassen kein Mittel zur Beschleunigung der Geburt unangewandt (z. B. Bad, Einlauf, Umhergehen, Kristeller, Morphiuminjection ets.), bis deutliche Zeichen auftreten, die eine stricte Indication entweder von Seiten der Mutter oder des Kindes stellen lassen. Die übrigen Indicationspunkte bedürfen hier wohl keiner weiteren Erwähnung, da sie ja allgemein bekannt und allgemein gültige sind.

Wenden wir uns nun zur Betrachtung des hiesigen Materials, so wurde zur Rettung des Kindes allein die Zangenoperation in 440 Fällen $=63,13$ pCt. vorgenommen. In 99 Fällen ging die Indication nur von Seiten der Mutter aus $=14,2 \mathrm{pCt}$., und in 158 Fällen $=22,67$ pCt. von beiden. Demnach wurde die Zange in den meisten Fällen zur Rettung des.Kindes angelegt, also wenn es sich handelte um Sinken oder Beschleunigung der kindlichen Herztöne unter 80-100 oder über 160, mit oder obne gleichzeitigen Abgang von Meconium, oder um Vorfall kleiner Theile.

Gaben Mutter und Kind die Indication, so handelte es sich meistentheils um Erschöpfung der Mutter mit gleichzeitiger Störung der kindlichen Circulation, oder um bohes Fieber der Mutter mit beginnender Asphyxie des Kindes; selbstverständlich finden sich darunter auch Fälle, wo die Indication von Seiten des Kindes docb noch eine dringendere war, als die der Mutter; da aber beide Theile betheiligt waren, so wurden sie dieser Rubrik eingefügt. 
692 Leisewitz, Die Zange in der Therapie des engen Beckens.

In den Fällen, wo die Indication nur von der Mutter ausging, handelte es sich um schwere Erkrankung derselben, Eklampsie, Nephritis, Urämie, incompensirte Herzfehler, Phthise, Erschöpfung und Wehenschwäche oder Wehenlosigkeit nach langer Geburtsdauer ohne bemerkbare Störung der kindlichen Circulation, oder um Ausbildung eines Contractionsringes, der rasch anstieg, oder um bestehende oder drohende Infection durch lange zurückliegenden Blasensprung, oder um Blutungen in Folge tief sitzender oder vorliegender Placenta.

Wie aber auch die Fälle im Einzelnen gelegen haben mögen, so kann man doch ruhig sagen, dass in der Mehrzabl aller Zangenentbindungen, wenn es sich nicht um Lebensbedrohung der Mutter handelte, die Geburt der Hauptsache nach beendigt wurde, um ein lebendes Kind zu erhalten. Denn, selbst wenn die Mutter allein erkrankt ist, ohne dass bereits Störungen von seiten des Kindes nachweisbar sind, so würden solche mit aller Sicherheit doch in nicht weiter Ferne eintreten, und das kindliche Leben in Mitleidenschaft gezogen werden.

Diese Erwägung bedingt nicht zum wenigsten die Anlegung des Forceps; selbst in solchen Fällen, wo ein schwerer Collaps der Mutter besteht, wollte man das kindliche Leben nicht preisgeben.

Ein Vergleich verschiedener Kliniken fübrte zu folgender Uebersicht:

Indication rom Indication von
Kinde
der Mutter

von beiden

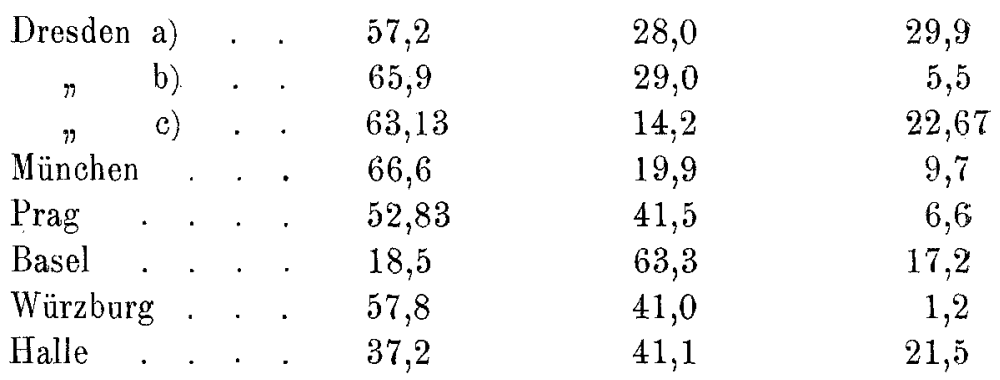

Hieraus ersieht man, dass die Zange meistens zur Rettung des kindlichen Lebens angelegt wird, dass aber in einigen Kliniken die Indication von seiten der Mutter überwiegt. Es muss aber hierbei, wie schon früher erwähnt, berücksichtigt werden, dass in verschiedenen Anstalten die Zange experimenti causa angelegt wird, 
ohne ganz zwingende Indication von seiten der Mutter oder des Kindes; was. in hiesiger Klinik nicht gestattet ist.

Betrachten wir nun die um des Kindes Willen erfolgten Zangenoperationen im Verhältniss zu den verschiedenen Beckenarten, so finden wir bei:

allgemein verengtem Becken . . . . 229 Fälle $=66,5$ pCt. normalem Becken. . . . . . . . . $109 n=78,9$ n plattem Becken . . . . . . . $47 "=60,2 "$ platt-rhachitischem Becken . . . . . . $39 "=65,6 n$ allgemein verengt platt-rhachitischem Becken $\frac{16 n}{460}=50,0$ "

Sieht man hier vorläufig von dem normalen Becken ab, wo andere Momente eine Rolle spielen, so ist bei allgemein verengtem Becken die höchste Zahl erreicht, d. b. in 66,5 pCt. musste zur Rettung des Kindes die Zange angelegt werden.

Die Erklärung liegt ja in der Fortsetzung der Beckenenge bis in den Ausgang und in der damit sich leicht steigernden Asphyxie des Kindes.

Stellt man demgegenüber die tiefe Aushöhlung des Kreuzbeins und den nicht wesentlich verengten oder manchmal sogar übernormal weiten Beckenausgang bei den 3 anderen Beckenformen, so fällt bei diesen die starke Compression fort, weshalb der Procentsatz der Zangen kein so hoher ist.

Die hohe Procentziffer bei dem normalen Becken beruht hauptsächlich darauf, dass es sich mehrmals um Vorfall oder Vorliegen der Nabelschnur und um grosse Kinder gehandelt hat, bei denen in Folge langer Geburtsdauer Störungen eingetreten sind.

Wie gross ist nun in den letzten 13 Jahren die Häufigkeit der Zangenoperationen im Beckenausgang, in Beckenmitte und im Beckeneingang gewesen, und wie verhalten sich dabei die 5 verschiedenen Beckenarten?

Von den 697 Frauen wurden:

bei Kopf im Beckenausgang . . . . . . . $310=44,5$ pCt. " " "Beckenmitte . . . . . . . . $350=50,21$ " und bei hochstehendem Kopfe (im Beckeneingang) $37=5,31$ " 697 der Zangenentbindung unterzogen.

Die hohe Zahl der 2. und 3. Gruppe zusammengenommen 
muss im ersten Augenblick auffallen, erklärt sich aber dadurch, dass in unserem Material jedes 4. Becken verengt ist.

Von den 310 Beckenausgangszangen treffen auf:

allgemein verengtes Becken . . . . $149(344)=43,31$ pCt. normales Becken. . . . . . . . $94(178)=52,81$ " plattes Becken . . . . . . . $32(78)=41,02$ " platt-rhachitisches Becken . . . . . $26(65)=40,0$ " allgemein verengt platt-rhachitisches Becken $9(32)=28,12$ "

$310(697)$

Die relative Häufigkeit bei normalem Becken beruht auf 14 Fällen von Eklampsie, auf 13 Fällen von tiefem Querstand, 3 Vorderhauptslagen und auf 2 Fällen von Wehenschwäche in Folge Scopolamininjection. Bei den übrigen Frauen handelte es sich zum Theil um grosse Kinder, zum Theil um vorzeitigen Blasensprung und dadurch bedingte Gefahr für Mutter und Kind, zum Theil um inficirte Frauen, zum Theil um eine unaufgeklärte Asphyxie der Kinder. Demnach ist also der Procentsatz der Zangen im Beckenausgang bei allgemein verengtem Becken doch ein absolut höherer als bei dem normalen Becken. Er steht mit 43,31 pCt. denen der übrigen 3 Beckenformen voran, was durch die schon erwähnte Verengerung des Beckenausgangs, speciell durch vorspringende Spinae, unbewegliches in das Becken vorspringendes Steissbein und spitzen Schambogen bedingt wird.

Anders verbält es sich bei den 350 Zangen in Beckenmitte, wo wir folgende Zusammenstellung finden:

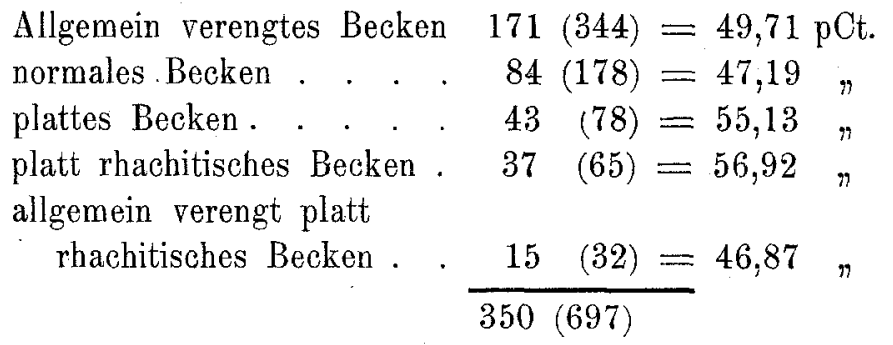

Hier nimmt der Procentsatz vom normalen Becken bis zum platt rhachitischen zu. Bei dem allgemein verengt platt rhachitischen Becken ist er wieder ein niedriger. Das hat seinen Grund darin, dass im Allgemeinen bei dem allgemein verengt platt rhachitischen Becken die Geburten, falls überhaupt nöthig, weniger durch Zange, als entweder durch Perforation oder durch Symphyseotomie bezw. Hebotomie oder Sectio caesarea conserv. aut Porro beendet worden 
sind, und die Zahl der allgemein verengt platt rhachitischen Becken überhaupt keine so grosse ist.

Im Verhältniss der zunehmenden Verengerung des Beckens nimmt hier der Procentsatz der Zangenanlegung eberfalls zu. Beim allgemein verengten Becken mit dem Typus des Trichterbeckens, wo der Beckeneingang mit der günstigen Kopfeinstellung (Hinterhaupt tiefer) noch ein ziemlich weiter ist (- $10 \mathrm{~cm}$ Diag. 8 Vera), tritt der kindliche Schädel bei kräftigen Wehen doch noch häufiger über die Beckenmitte hinaus, ohne dass die Einwirkung der Compression schon eine gefährliche ist. Oder die Compression im Beckeneingang ist noch keine so starke, dass das Kind sich nicht beim Tiefertreten des Kopfes wieder etwas erholen kann. Dagegen bei dem platten und platt-rhachitischen Becken, wo wir Verengerungen im Beckeneingang - $9 \mathrm{~cm}$ Diag. - $7 \mathrm{~cm}$ Vera finden, kann die Compression im Beckeneingang sohon eine so erhebliche sein, dass die Circulationsstörungen selbst in der weiten Beckenhöhle sich nicht mehr in kurzer Zeit wieder beheben können. Daher begegnet man bei diesen beiden Formen auch dem höheren Procentsatz der Zangen, nämlich 55 resp. 57 pCt. Bei dem allgemein verengt platt rhachitischen Becken mag vielleicht der wieder etwas erweiterte Beckeneingang ( $-9,5-7,5)$ eine Rolle spielen, so dass die Zahl der in Beckenmitte angelegten Zangen im Verhältnis wieder eine geringere ist $(46,87 \mathrm{pCt}$.).

Was endlich die hohe Zange anlangt, so verfügen wir in den 13 Jahren nur über eine geringe Anzahl, nämlich im Ganzen 37 $(697)=5,31$ pCt., so dass auf ein klinisches Jahr ca. 3 kommen. Sie vertheilen sich auf das

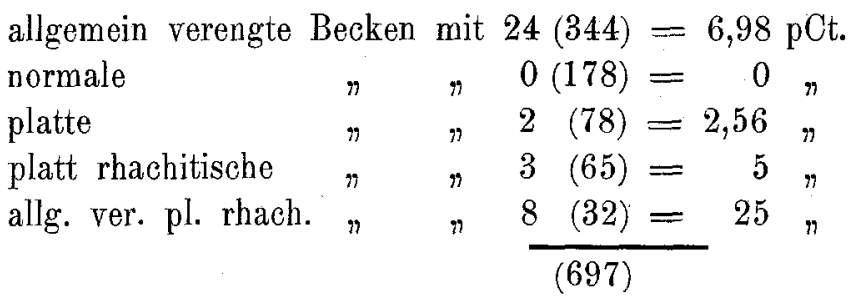

Daraus ersieht man, dass der Procentsatz vom platten Becken zum platt rhachitischen und dann zum allgemein verengten bis zum allgemein verengten platt rhachitischen Becken immer steigt. Und wenn wir uns fragen, warum bei dem allgemein verengten Becken mit einer Diagonalis von $10 \mathrm{~cm}$ doch im Verhältniss so viele hohe Zangen gemacht worden sind, so liegt die Antwort 
wieder in dem Missverhältniss zwischen Becken und kindlichem Schädel, und fast in allen Fällen handelte es sich um Rinder zwischen 3300 bis $4500 \mathrm{~g}$, bei denen also schon Störungen im Beckeneingang eintreten konnten oder deren Mütter durch die lange Geburtsdauer und theils fruchtlos wirkende Wehen geschwächt waren.

Ebenso findet sich bei dem allgemein verengt platt rhachitischen Becken ein Missverhältniss zwischen Kind und Becken, welches den relativ hohen Procentsatz von 25 zur Genüge erklärt.

Endlich muss noch erwähnt werden, dass auch die straffen Weichtheile der I. Gebärenden eine nicht zu unterschätzende Rolle mitspielen. Es stehen 551 Primiparae $=79,05$ pCt. 146 Multiparae $=20,94$ pCt. gegenüber.

Was nun endlich die Prognose der Zangenoperationen betrifft, so interessiren uns die Todesfälle bezw. die Verletzungen der Mutter und der Kinder. Im besonderen muss die Mortalitätsziffer bei den verschiedenen Beckenarten und bei typischer und atypischer Zange hervorgehoben werden.

Die Hauptaufgabe aber haben wir in der Epikrise zu erblicken, ob eine Reihe von Todesfällen der Kinder, die nicht der Technik zur Last fallen, durch eine zweckmässigere Geburtsleitung bezw. Anzeigestellung zu vermeiden gewesen wären.

Die Gesammtmortalität der Mütter betrug 21 von $697=$ $3,01 \mathrm{pCt}$. Von diesen entfallen aber auf Tod durch Eklampsie 11, in Folge von anderen Erkrankungen (Nephritis, Herzfehlern, Pneumonie) 5 und in Folge von Sepsis ebenfalls 5. Von diesen letzteren kommt ein tödtlicher Ausgang auf einen Fall $v o n$ Uterusruptur, welcher schliesslich mit der Zange entbunden wurde. Es sind demnach 17 Fälle für die Zange in Abrechnung zu bringen, so dass auf 680 Zangen 4 Todesfälle kommen, welche der Klinik zur Last fallen $=0,58 \mathrm{pCt}$. Mortalität.

Mit den Ergebnissen einzelner Kliniken (aus der Statistik im Winckel'schen Handbuch) verglichen, nimmt dann die unsere die drittniedrigste Ziffer ein:

\begin{tabular}{|c|c|c|c|c|}
\hline Münchmeye & & 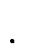 & $(1883-1888)$ & $3,4(0,0)$ \\
\hline Wahl & . & - & $(1889-1904)$ & $5,2(0,0)$ \\
\hline Verf. & • & . & $(1894-1906)$ & 0,58 \\
\hline Winckel. & . & . & $(1883-1902)$ & 0,47 \\
\hline Collin & . & . & & 0,3 \\
\hline Chrobak. & & . & & 1,38 \\
\hline Sehauta. & . & . & & 1,5 \\
\hline Schick & . & . & $(1891-1894)$ & 4,7 \\
\hline
\end{tabular}


Die Gesammtmortalität der Kinder betrug 109 (697) $=15,63$ pCt.; davon entfallen auf Schuld der Zange (Fractur, Compression) $33=4,73$ pCt.; auf Tod durch schwere, post partum nicht mehr zu behebende Asphyxie und auf Exitus intra partum in Folge Asphyxie $46=6,59$ pCt., ferner auf Tod in Folge angeborener Fehler (Missbildungen) oder späterer Krankheiten (Lues, Arteriitis umbilicalis, Magendarmkatarrh, Pneumonie) $30=4,3 \mathrm{pCt}$.

Die erstgenannten Zahlen bedürfen noch einer weiteren $\mathrm{Be}$ sprechung, und zwar vom Gesichtspunkt der Indication aus. Von den 3 Hauptindicationen: Mutter, Mutter und Kind, Kind allein kommen hier nur die beiden letzten in Erwägung, denn bei diesen beiden Gruppen soll das in Gefahr befindliche Kind gerettet werden.

Verfolgt man zunächst die 440 Fälle mit Indication von Seiten des Kindes, so sind von diesen Kindern gestorben in Folge:

Fractur oder Compression . . 15 Kinder Asphyxie . . . . . . . 32 " Krankheit

demnach lebend $377=85,68$ pCt.

zusammen 63 Kinder; es bleiben

Da aber die an Krankheit später gestorbenen Kinder bei der Besprechung der Zangenmortalität nicht mitgerechnet werden dürfen, so sind 393 lebende Kinder $=89,32 \mathrm{pCt}$. zu verzeichnen.

In 158 Fällen bildeten Mutter und Kind die Indication zur Zangenoperation; von diesen sind gestorben in Folge

Fractur oder Compression . . 8 Kinder

Asphyxie . . . . . . . 14 "

Krankheit

demnach lebend $129=81,64 \mathrm{pCt}$.

zusammen 29 Kinder; es bleiben

In Abzug kommen wieder die an Krankheit verstorbenen; somit ändern sich die Zablen auf $136-86,07$ pCt.

Nach Zusammenstellung beider Gruppen ergiebt sich folgendes: In 598 Fällen wurde die Zange angelegt, um das kindliche Leben zu retten (inbegriffen die Fälle, in denen neben dem Kinde auch die Mutter die Zange indicirt erscheinen liess), von diesen bleiben $529=88,46$ pCt. lebend, es starben also (die an einer Krankheit später erlegenen Kinder abgerechnet) 11,54 pCt.

Vergleicht man andere Statistiken, so lauten auch hier unsere Zahlen nieht ungünstig: 
698 Leisewitz, Die Zange in der Therapie des engen Beckens.

\begin{tabular}{|c|c|c|c|}
\hline Münchmeye & & $(1883-1888)$ & 13,4 \\
\hline Wahl & & $(1889-1894)$ & 21,2 \\
\hline Verf. & & $(1894-1906)$ & 11,54 \\
\hline Winckel. & & $(1883-1902)$ & 15,5 \\
\hline Collin & & & 23,8 \\
\hline Chrobak. & & & 23,62 \\
\hline Schauta. & & & 4,6 \\
\hline Sehick & & $(1891-1894)$ & 11,3 \\
\hline
\end{tabular}

Angesichts dieser Zahlen muss man nun sagen, ob es nicht möglich 'gewesen wäre, die an Asphyxie gestorbenen Kinder doch noch durch frühzeitigere Anlegung der Zange zu retten?

Bei der Besprechung der beiden Gruppen (Zange nach Indication von Seiten des Kindes und von Seiten der Mutter und des Kindes) fanden wir, dass im Ganzen 46 Kinder an Asphyxie zu Grunde gegangen waren, also allein 7,69 pCt.. Das ist allerdings ein ziemlich hoher Procentsatz. Allein auch hier müssen selbstredend einige Fälle in Abrechnung gebracht werden und zwar diejenigen, bei denen es sich um Nabelschnurvorfall (6) und mehrfache Nabelschnurumschlingung (2) handelte; demnach kommen 8 in Abzug $=38$ und der Procentsatz sinkt auf $6,35 \mathrm{pCt}$.

Aber auch dieser erscheint uns noch viel zu hoch. Er wird sich aber besser beurtheilen lassen, wenn, wie im Folgenden geschehen soll, erläutert wird, auf welche Beckenarten und auf welchen Kopfstand die kindlichen Todesfälle entfallen:
1. allgemein ver- engtes Becken
a) Hohe Zange.
$\left.8(24)^{1}\right)=33,33 \mathrm{pCt}$
b) Zange in Beckenmitte
$21(171)=12,29$ \%
c) Zange im Beckenausgang
$12(149)=8,05 \%$

2. normales Becken

a) Hohe Zange :

b) Zange in Beckenmitte

$12(84)=14,28 \mathrm{pCt}$.

c) Zange im Beckenausgang

$(94)=4,25 \%$

3. plattes, Becken

a) Hohe Zange . . . . . . 0

b) Zange in Beckenmitte . 8

$(2)=0 \mathrm{pCt}$.

c) Zange im Beckenausgang

$(43)=18,6$,

4. platt-rhachit. Becken

a) Hohe Zange . . . . . . 0

b) Zange in Beckenmitte $\cdot \cdot 6$

$(32)=3,12 \%$

5. allgemein verengtes platt-rha-

c) Zange im Becisenausgang

(3) $=0 \mathrm{pCt}$.

$(37)=16,21$

$1(26)=3,85 \%$ chitisches Becken

a) Hohe Zange .

b) Zange in Beckenmitte

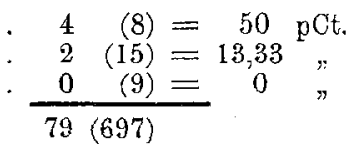

1) (Die eingeklammerten Zahlen bedeuten die Anzahl der Zangen.) 
Vergleichen wir zuerst die Ergebnisse der hohen Zange, so finden sich Todesfälle der Kinder nur beim allgemein verengten und allgemein verengt platt-rhachitischen Becken (bei normalem Becken wurden überhaupt keine hohen Zangen angelegt), im ersten Falle in 33 pCt., im 2. in 50 pCt. Daraus geht dentlich hervor, dass die hohe Zange bei diesen beiden Beckenarten, wo es sich nicht bloss um einen verengten Beckeneingang handelt, sondern auch um eine enge Beckenhöhle, eine äusserst schwierige und gefährliche Operation ist, die absolut nicht die Aussichten bietet, in deren Erwartung man die Operation vornimmt. Theilweise ist der kindliche Organismus schon derartig alterirt, um Störungen in der Hirnfunction oder directen Blutaustritt hervorzurufen; anderentheils ist es nur durch eine starke Traction im Zusammenhang mit Compression möglich, den nicht oder nur gering configurirten Kopf der Beckenhöhle anzupassen, was natürlich bei dem bestehenden Missverhältniss zwischen Kopf und Becken zu todtbringenden Veränderungen führen muss. Aus diesem Grunde muss an Stelle der hohen Zange, wie schon erwähnt, eine andere Operation treten, nämlich die Hebotomie bei günstigen mütterlichen und kindlichen Verhältnissen oder die Perforation selbst des lebenden bezw. absterbenden Kindes mit dem Rückhalt, dass bei der nächsten Schwangerschaft die künstliche Frühgeburt oder die Hebotomie bezw. Sectio caesarea conservativa in Erwägung gezogen werden muss.

Die Ergebnisse der Zangen in Beckenmitte (mit Ausnahme des normalen Beckens) führen zu ähnlichen Schlüssen. Den höchsten Procentsatz finden wir bei dem platten und platt-rhachitischen Becken; dann erst folgen allgemein verengt platt-rhachitisches und allgemein verengtes Becken. Diese Reihenfolge bat wohl ihren Grund darin, dass bei den beiden erstgenannten Beckenarten durch ein langes Verweilen des Kopfes im Beckeneingang zwecks Configuration der langdauernde Druck genügt hat, um die Asphyxie des Kindes herbeizuführen. Obwohl sich der Kopf in der geräumigen Beckenmitte befindet, so kann sich der kindliche Organismus doch nicht mehr erholen. Selbst die an sich nicht schwere Zangenextraction kommt zu spät, um das Kind noch zu retten. Man beobachtet ja sehr häufig, dass der Kopf, nachdem er lange im Beckeneingang gestanden hat, förmlich durch die übrigen Beckenebenen hindurchstürzt und die Geburt rasch vollendet ist. Wenn aber Wehenschwäche in Folge der langen Geburtsarbeit bei im Beckeneingang stebenden Kopfe eintritt, dann bleibt der Kopf auch 
in Beckenmitte noch stehen, und es wird die Asphyxie erhöht und schliesslich letal.

Bei allgemein verengt platt-rhachitischem und allgemein verengtem Becken genügt der fortdauernde Druck trotz der guten Configuration des Kopfes bis in Beckenmitte, um die Asphyxie zu erhöhen, und es bedarf genauer Abwägung und guter Beobachtung, wenn man den richtigen Zeitpunkt treffen will, um noch ein lebendes Kind mit der Zange zu erhalten. Hiermit erklärt es sich, dass bei der ersten Beckenart häufiger Todesfälle vorkommen, da die Beckenhöhle eine engere ist als bei der zweiten Form.

Die Zahl der Todesfälle bei normalem Becken erfordert eine besondere Erläuterung wegen des hohen Procentsatzes von 14,28. Bei den 12 Todesfällen handelte es sich 4 mal um sehr grosse Kinder (über $50 \mathrm{~cm}$ lang und 3900 bis zu $5200 \mathrm{~g}$ schwer); in 1 Fall um Nabelschnurvorfall, in 2 Fällen um Vorderhauptslage. Somit sind 7 Todesfälle durch die Lage der Dinge entschuldbar. Nur in 5 Fällen liegt die Ursache in der mangelhaften Technik $=5,95$ pCt., demnach im Vergleich zu den anderen Ergebnissen bei in Beckenmitte angelegter Zange der erwartete geringste Procentsatz.

Was die typischen Beckenausgangszangen anlangt, so finden sich nur niedrige Procentsätze aufgezeichnet, unter denen die erste Stelle wieder das allgemein verengte Becken mit 8,05 pCt. einnimmt; hier spielt aber der verengte Beckenausgang die Hauptrolle. Durch ihn wird die Extraction sehr erschwert, auch wird häufig wohl zu lange mit der Anlegung gewartet, da man bei dem fast sichtbaren Kopf unter der Wirkung des Kristeller'schen Handgriffes die Spontangeburt noch erhofft.

Schliesslich müssen noch die Verletzungen der Mutter und des Kindes besprochen werden.

Rechnet man jede kleinste Verletzung, die genäht werden musste, mit, so finden sich unter den 697 Zangenoperationen 513 Verletzungen der Mutter $=73,6$ pCt.; unverletzt blieben 189 Erauen $=27,11$ pCt.; die Verwundungen vertheilen sich auf Scheidendammriss $(279 \mathrm{mal})=40,03$ pCt., completen Scheidendammriss $(6)=0,86$ pCt., Collumriss $(32)=4,59$ pCt., Episiotomien $(184)=26,39$ pCt., Collumincisionen (11), Dammincisionen 1; ausserdem muss eine Blasenscheidenfistel erwähnt werden. 
Im Vergleich zu unseren früheren Aufzeichnungen und aus anderen Kliniken ist ein wesentlicher Unterschied nicht zu bemerken.

Münchmeyer berichtete über $85,0 \mathrm{pCt}$. Verletzungen

$\begin{array}{lccccc}\text { Wahl } & & & 81,4 & & \\ \text { Verf. in den letzten } & \text { 13 Jahren } & 73,6 & & \\ \text { Schmid } & \text { berichtete über } & 84,6 & & n \\ \text { Schick } & n & n & 60,0 & & \\ \text { v. Winckel } & " & n & 29,0 & n & " \\ \text { Chrobak } & " & n & 80,0 & & \end{array}$

Von Verletzungen des Kindes sind hervorzuheben:

in 38 Fällen Facialislähmung $=5,45 \mathrm{pCt}$.

in 8 Plexuslähmung $=1,15$,

Fassen wir alle in dieser Arbeit gewonnenen Resultate zusammen, so kommen wir zu dem Schluss, dass bei äusserst exacter Indicationsstellung und vorsichtigem Verfahren, trotz der Unmenge pathologischer Becken, die Zange zu der gewünschten Rettung des kindlichen Lebens führte, freilich mit sehr vielen Verletzungen der Mütter. In Folge dessen bleibt für unsere Klinik auch heute noch der Satz zu Recht bestehen, dass die Zange namentlich beim engen Becken eine für die Mutter gleichgültige Operation durchaus nicht ist und dass ihre Anzeigestellung erst recht in der Privatpraxis sehr genau genommen werden muss.

Andererseits darf aber auch nicht verkannt werden, dass in einzelnen unserer Fälle (bei Asphyxie etc.) die Zange noch früher hätte angelegt werden sollen, um das kindliche Leben zu retten.

Endlich ist die hohe Zange unbedingt auszuschliessen, namentlich für den praktischen Geburtshelfer, und an ihre Stelle die weniger schädigende Operation zu setzen, die Hebotomie.

Ist diese aber für den praktischen Geburtshelfer im einzelnen Falle weder durchführbar, noch rathsam, so zögere er nicht zu perforiren und sei es auch das noch lebende Kind. 